Ute Günkel-Maschek

\title{
In die Augen, in den Sinn. Wandbilder als konstitutive Elemente von (Handlungs-)Räumen in der minoischen >Neupalastzeit
}

This paper analyses the interaction between wall paintings, places and spaces (of activity) in the Minoan Neopalatial and Final Palatial Period on the theoretical basis of a »Pictorial-Space « concept. In the case of the palace at Knossos there are at least three types of spaces, each distinguished by a specific type of painting: entryways were frequently decorated with a bull motif, passageways with processions, and in the sthrone room < both the throne and a door in the rearward wall were flanked by griffins which provided a backdrop for the throne and created the impression of a kind of >door of epiphany.< The paintings therefore functioned either as an emblem that made symbolic reference to the ruler for those entering the palace, or, as in the case of the passageway, they presented idealized depictions of activities that were actually performed in the space in question, or, as in the case of the throne-room griffins, they lent a complementary layer of meaning to events that took place onsite, presenting the person who entered the sthrone room< and sat on the throne as the epiphany of a divinity.

Die Bildwerke der kretisch-bronzezeitlichen Kultur geben ein faszinierendes, wenngleich in seinem fragmentarischen Erhaltungszustand oft frustrierendes Zeugnis von der Verwendung visueller Medien, mittels derer Inhalte und Bedeutungen in bestimmten Kontexten präsentiert und kommuniziert wurden. Zur Bebilderung der minoischen Lebenswelt griff man auf ein breites Spektrum an materiellen Bildträgern, Themen und Motiven zurück, die je nach Anlass, Kontext und beabsichtigter Wirkung zum Einsatz gebracht wurden. Das Wandbild, die monumentalste Form minoischer Bildwerke, gehörte zu den eindrucksvollsten Mitteln, um architektonische Räume zu dekorieren und ihnen repräsentative oder symbolische Konnotationen zu verleihen. Durch Wandbilder wurden indes nicht nur einzelne Bereiche oder ganze Räume gegenüber undekorierten Bereichen und Räumen hervorgehoben. Vielmehr wurden Wandbilder in Hinblick auf ihre Wahrnehmung durch im architektonischen Raum agierende Personen gewählt, komponiert und platziert, somit dienten sie der visuellen Inszenierung und inhaltlichen Prägung architektonisch eingefasster Handlungsräume ${ }^{1}$. In diesem Sinne können sie neben architektonischen Strukturen und Kleinfunden als eine weitere materielle Quelle für die Erschließung von Raumfunktionen und -nutzungen herangezogen werden sowie, mehr noch, für die visuelle Akzentuierung jener Funktionen und Nutzungen durch Blick lenkende und Inhalt vermittelnde Gestaltungselemente.

Minoische Wandbilder stammen überwiegend aus architektonischen Kontexten, die durch »palatiale $\ll^{2}$ Elemente geprägt sind, d. h. aus den so genannten Palästen selbst sowie

1 Cameron 1970, 163-166; Cameron 1978, 580; Blakolmer 1995, 463-464; Blakolmer 2000, 397. s. ferner Palyvou 1987, 195 .

2 Ich folge hierin Blakolmer 1995, 467 Anm. 28: »Der Terminus >palatial< bezeichnet hier eine innovative Architekturform und nicht die Funktion eines Gebäudes«. 
aus den Stadthäusern in Knossos und den über die Insel verteilten >Villen<, die im Besitz einer herrschenden bzw. elitären Gesellschaftsschicht gewesen sein dürften. Neben dem gemeinsamen architektonischen >Vokabular legen die nicht nur in den Wandbildern, sondern auch bei zahlreichen weiteren Bildwerken verwendete Motivik und Symbolik nahe, dass sowohl das Geschehen in den >Villen und Stadthäusern als auch jenes in den >Palästen $<$ mit ähnlichen Ideen und Anschauungen verknüpft war. Während in den >Villen $<$ und Stadthäusern jedoch oftmals nur einzelne Räume farblichen Raumdekor erhielten, waren es im Palast von Knossos Eingangsbereiche, Durchgangsräume und `Aufenthaltsräume<, an deren Wänden die mit dem Geschehen im Palast und dem Herrschaftsanspruch der Elite assoziierten Ideen und Vorstellungen durch Bilder präsentiert und kommuniziert wurden ${ }^{3}$. Zur Annäherung an jenes Phänomen der durch Wandbilder geprägten (Handlungs-)Räume soll in diesem Beitrag nach einer Skizzierung der theoretischen und methodischen Überlegungen in drei Erklärungshypothesen vorgestellt werden, auf welche Weise die Wandbilder in ausgewählten Bereichen des endpalastzeitlichen Palastes von Knossos (Spätminoisch [SM] II - IIIA1, ca. 1490-1370 v. Chr.) 4 in Hinblick auf das dortige Handlungsgeschehen gewirkt haben könnten.

\section{Bild-Raum und Handeln - Theoretische und methodische Überlegungen}

Der Raum als konstitutive Dimension von Lebenswelten ist in den letzten Jahrzehnten verstärkt in den Mittelpunkt des wissenschaftlichen Interesses gerückt. Vor allem in den Sozialwissenschaften wird Raum dabei nicht mehr nur als Rahmenbedingung des menschlichen Handelns zur Kenntnis genommen. Dem konstruktivistischen Raumbegriff Martina Löws zufolge wird Raum in Handlungs- und Platzierungsprozessen sowie in der synthetisierenden Wahrnehmung von platzierten Lebewesen und Objekten durch das Individuum konstituiert ${ }^{5}$. Raum ist demzufolge kein in erster Linie materielles Gebilde mehr, sondern lässt sich als relationale (An-)Ordnung von sozialen Gütern und Menschen an Orten begreifen. Durch die temporäre oder dauernde Platzierung von Gestaltungselementen werden räumliche Arrangements an Orten fixiert. Die materielle Gestaltung von Orten ist dabei bestimmend für deren soziale Wirkung und beeinflusst aufgrund von kollektiv attribuierten Bedeutungen und Wertigkeiten das Raum konstituierende Handeln und Verhalten ${ }^{6}$.

$3 \mathrm{Zu}$ Wandmalereiprogrammen in minoischen Palästen siehe vor allem Hägg 1985; Cameron 1987; Marinatos 1996.

4 Momigliano 2007, 7 Taf. O, 2. Zu dieser Phase unter Betonung deren nicht-mykenischen Charakters

S. Niemeier 1983 .

5 Löw 2001, 152-230.

6 Löw 2001, 198; Schroer 2006, 176-178. 
Raum impliziert in diesem Sinne also zweierlei: Zum einen besteht er als >Handlungsraum < in den relationalen (An-)Ordnungen, die in Handeln und Wahrnehmung zwischen Lebewesen und Objekten hergestellt werden. Die Platzierung der Lebewesen und Objekte sowie durch deren Aussehen und Materialität evozierte symbolische Konnotationen prägen das physisch-räumliche Erscheinungsbild und beeinflussen den Prozess der Raumkonstitution sowie das Handeln und Verhalten jedes einzelnen. Zum anderen lässt sich Raum als kollektive Sichtweise relationaler Gefüge begreifen, welche als in sozialen Prozessen angeeignete Vorstellungen räumlicher Arrangements vorhanden sind und an den dafür vorgesehenen Orten im Handeln reproduziert werden. Die materielle und symbolische Gestaltung von Orten ist darauf angelegt, an mit den Gestaltungselementen verknüpfte Vorstellungen zu appellieren und ein bestimmtes Handeln und Verhalten zu bewirken. Wenn im Folgenden also von »(Handlungs-)Räumen« die Rede ist, so soll damit jene duale Qualität des Raums hervorgehoben werden, die die gegenseitige Abhängigkeit von gestalteten Orten und menschlichem Handeln bedingt.

Das Potential eines solchen Raumbegriffs für die Archäologie besteht darin, architektonische Räume nicht mehr nur als kontextdeterminierende Einheiten zu begreifen, sondern als Orte, die durch die gezielte Herstellung materieller Strukturen und die Aufladung mit konnotativen Wertigkeiten als Orte ganz bestimmten sozialen Handelns inszeniert wurden. Nicht nur die hierfür genutzten Instrumentarien zeichnen sich bis zu einem gewissen Grad in archäologischen Befunden ab. Auch die Art der Handlung und damit zusammenhängende Bewegungsmuster und Blickrichtungen lassen sich ansatzweise rekonstruieren. Durch die Form und Materialität der architektonischen Elemente, die Einrichtung mit fixen oder mobilen Objekten sowie durch weitere Gebrauchsgegenstände erhielten die Räume funktionale und symbolische Bedeutungen, die das Handeln und Verhalten der Menschen prägten.

Eine wesentliche Sinnkomponente erhielten Orte durch die Anbringung, Aufstellung oder Verwendung von Bildwerken. (Handlungs-)Räume, die durch die Präsenz oder Verwendung von Bildwerken geprägt sind, sollen im Folgenden als »Bild-Räume« beschrieben werden7. In Bild-Räumen werden nicht nur durch bildliche Darstellungen Bedeutungsinhalte vergegenwärtigt und kommuniziert, die für oder im Bezug auf das Handlungsgeschehen relevant waren. In Bild-Räumen gehen auch die artifiziell präsenten Lebewesen und Objekte sowie die durch ihre Relationen zum Ausdruck gebrachten Inhalte in die Raumkonstitution ein und werden zu festen Bestandteilen der an jenen Orten lokalisierten (Handlungs-)Räume. Auf der Grundlage des Bild-Raum-Konzepts soll daher untersucht werden, in welchen räumlichen Kontexten Bilder verwendet wurden und welche Themen und Motive wie und in welchen Handlungskontexten vergegenwär- 
tigt wurden. Ziel ist es, der Verwendung von Bildern als Sinn konstituierenden Bestandteilen von (Handlungs-)Räumen näherzukommen.

Jedes Bildwerk kann auf diese Weise als Bestandteil von (Handlungs-)Räumen betrachtet und analysiert werden ${ }^{8}$. Die Gattung der Wandbilder, um die es hier geht, stellt in Hinblick auf die minoische Kultur das monumentalste Mittel der visuellen Kommunikation von Bedeutungen dar. Der Inhalt der Wandbilder sowie ihre Platzierung innerhalb des architekturräumlichen Gefüges dienten dazu, Bedeutungsgehalte an bestimmten Orten, für bestimmte Personen und in Hinblick auf bestimmte Handlungen zu vergegenwärtigen. Im Unterschied zu mobilen Bildwerken, deren Fundumstände nur in Einzelfällen Schlüsse auf ihre einstige Nutzung zulassen oder deren Verwendung oftmals nicht an heute noch feststellbare Kontexte gebunden war, bieten in situ gefundene Wandbilder trotz ihres meist fragmentarischen Erhaltungszustandes die Möglichkeit, bestimmte Themen und Motive in eindeutigen Bezug zu architektonisch gestalteten Orten und dort lokalisierbare, wenn auch nicht eindeutig fassbare Handlungen zu setzen. Je nach Funktion und Nutzung variierten die bildlichen Darstellungen und verliehen dem architektonischen Raum eine jeweils angemessene thematische oder symbolische Konnotation. Wandbilder bieten in dieser Hinsicht eine seltene Möglichkeit, sich - freilich vorbehaltlich chronologischer oder fundkontextbezogener Unsicherheiten - von archäologischer Seite der Konstruktion und Sinnstruktur einer materiellen, mit Bedeutung aufgeladenen Umwelt zu nähern. Auch wenn es bislang kaum möglich ist, die mit den bildlichen Darstellungen zum Ausdruck gebrachten Inhalte nachzuvollziehen, so sind doch die Bildobjekte, die dargestellten Menschen und Gegenstände in ihrem Zusammenhang als solche erkennbar und können als Mittel der Thematisierung und Kommunikation entsprechender Ideen am jeweiligen Ort begriffen werden.

Für eine Annäherung an minoische Bild-Räume sind daher neben den materiellräumlichen Gegebenheiten formale Aspekte und Themen des bildlichen Wanddekors einzubeziehen, die in Abhängigkeit von der architektonischen Struktur und der beabsichtigten Wirkweise der Bildthemen gewählt und angebracht wurden. Flächiger, großformatiger Wanddekor, einzelne Wandpaneele, auf Augenhöhe verlaufende horizontale Bildstreifen oder szenenreiche Miniaturdarstellungen im Wandbereich oberhalb der Türstürze dien-

8 Neben den Wandbildern lassen sich als prägende Elemente von Bild-Räumen auch dreidimensionale Bildwerke nennen, wie in Naturheiligtümern aufgestellte Terrakotten und Bronzefigurinen sowie in rituellen Akten zum Einsatz gebrachte Rhyta in Form von Tierköpfen, oder zweidimensionale Darstellungen auf in performativen, rituellen oder administrativen Akten zur Geltung kommenden, funktionalen Bildträgern, wie Siegelringe oder Gefäße mit Reliefdekor. Durch ihre bildliche Darstellung vergegenwärtigen beispielsweise Figurinen mit charakteristischen Gesten den dieser Haltung entsprechenden oder den mit dieser Handlung implizierten Sinn an dem Ort, wo jener Relevanz besaß. Tierkopfrhyta, die vermutlich bei Libationen zum Einsatz kamen, bringen jene Riten in Zusammenhang mit den Ideen, die mit den jeweiligen Tiermotiven assoziiert waren. 
ten sowohl wahrnehmungstechnisch als auch hinsichtlich der darstellbaren Bildinhalte unterschiedlichen Zwecken. Die bildliche Darstellung selbst ist häufig nur in fragmentarischen Ausschnitten erhalten und kann, wenn überhaupt, lediglich in Hinblick auf das Oberthema bestimmt werden, welches zur konnotativen Charakterisierung des (Handlungs-)Raums gewählt wurde. Vergleiche mit den erhaltenen Fragmenten in ähnlichen architektur- und handlungsräumlichen Kontexten sowie ikonographisch-vergleichende Analysen mit verwandten Darstellungen in anderen Bildgattungen können hilfreich sein, um Aspekte des Darstellungsinhalts oder zumindest des größeren Sinnzusammenhangs des ursprünglichen Wandbildes konkreter zu benennen. Als Nebeneffekt schließlich lassen sich Bildobjekte, die auch auf anderen Bildträgern vorkommen, mit dem Geschehen an existierenden Orten in Zusammenhang bringen.

Die Verschränkung von räumlichem Arrangement, bildlicher Darstellung und rekonstruiertem Handeln ermöglicht es also, Aspekte von Bild-Räumen zu fassen, wie es sie am jeweiligen Ort zumindest temporär gegeben haben könnte. Angesichts der Tatsache, dass keine erhellenden Texte zum Geschehen in den Räumlichkeiten der Paläste und anderer Gebäude zur Verfügung stehen, gewinnt dieser Ansatz umso mehr an Relevanz. Mit Bild-Räumen lassen sich demnach ganz besondere, wenn auch bedauerlicherweise ganz besonders rare Einblicke in die kulturell und materiell konstruierte Lebenswelt der bronzezeitlichen Kreter gewinnen.

Im Folgenden soll nun in der gebotenen Kürze das Bild-Raum-Konzept in seiner methodischen Praxis anhand dreier Arten von Bild-Räumen exemplifiziert werden, die im endpalastzeitlichen Palast von Knossos existierten (Abb. 1): der am Westhof gelegene Südwesteingang als Eingangsraum, der daran anschließende >Prozessionskorridor< als Beispiel für Durchgangsräume und schließlich der im Herzen des Palastes gelegene Thronraum als >Aufenthaltsraum<, als Ort des temporären Aufenthalts und Handelns. Die Wandbilder dieser drei Bereiche waren zum Zeitpunkt der Ausgrabungen zumindest in Resten in situ an den Wänden erhalten. Ihre Einordnung in einen architektonischen Kontext kann demnach als gesichert gelten. Hinsichtlich der Datierungen ist anzumerken, dass in keinem Fall eine eindeutige Festlegung des Zeitpunkts ihrer Anbringung gegeben ist, jedoch alle drei Beispiele bis zum Ende des Palastes an den Wänden blieben und somit zumindest einige Jahrzehnte lang gleichzeitig als prägende Elemente von Handlungsräumen bestanden haben müssen. 


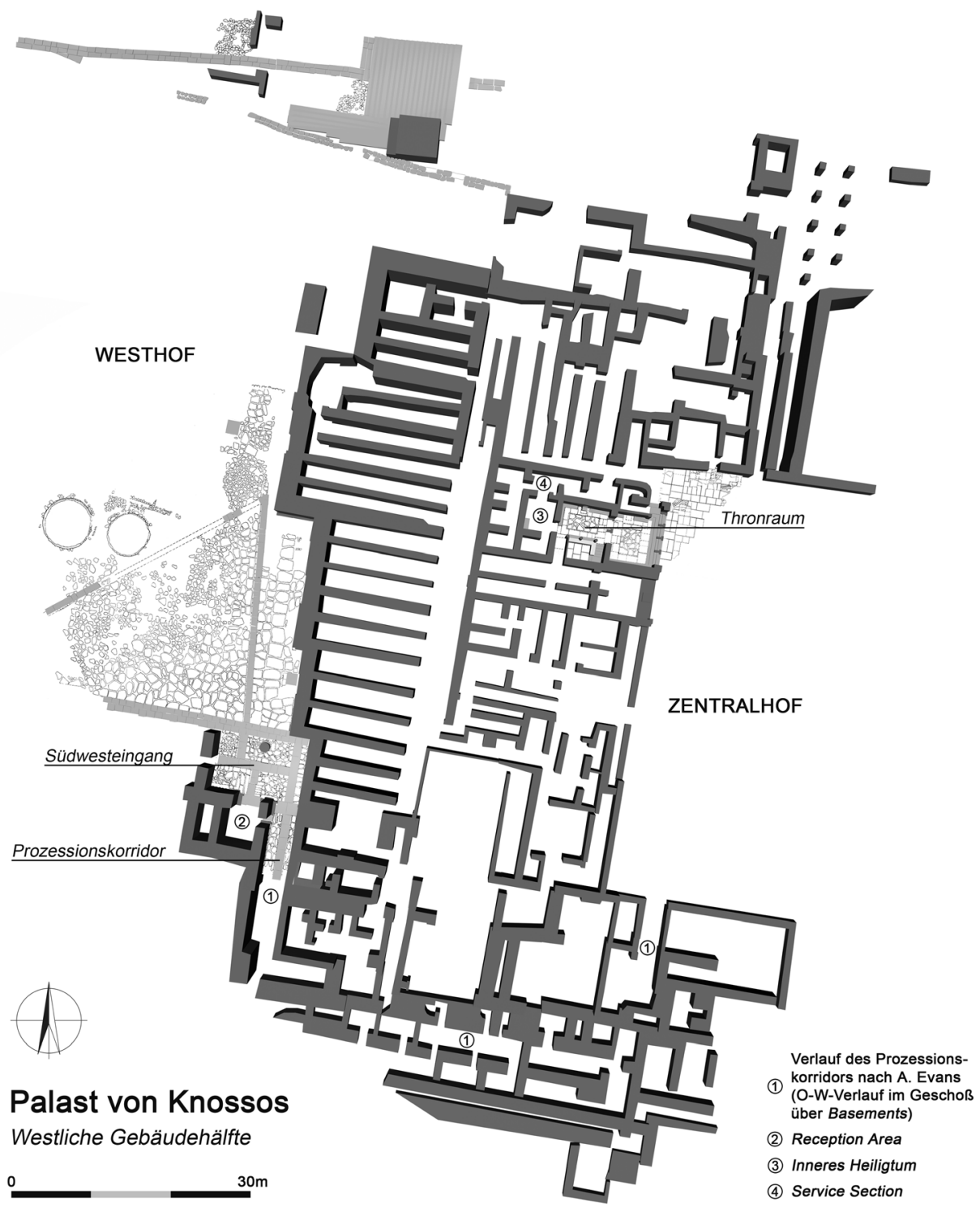

Abb. 1 | Grundriss der westlichen Gebäudehälfte des Palastes von Knossos (M. 1: 1000). 


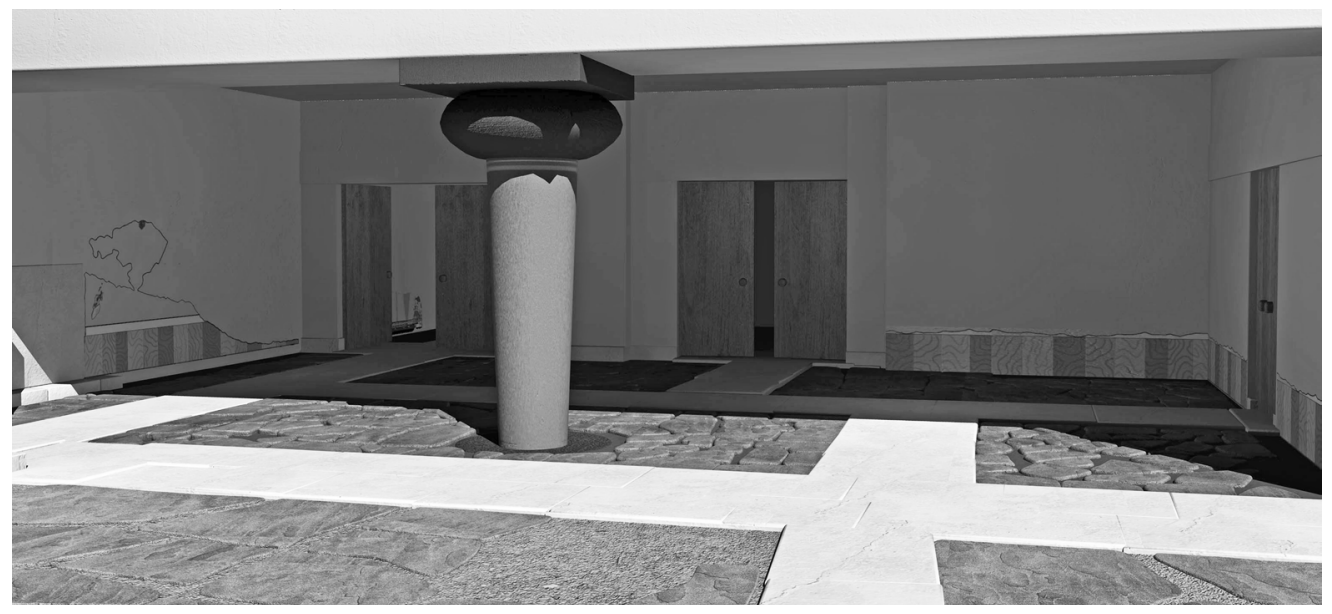

Abb. 2 | Südwesteingang und Beginn des >Prozessionskorridors<. Versuch einer Re-Platzierung der erhaltenen Wandmalereireste im architektonischen Kontext, Blick nach Süden.

\section{Bild-Räume im endpalastzeitlichen Knossos: drei Fallbeispiele}

\subsection{Der Südwesteingang in den Palast von Knossos}

Eingangsräume sind grundsätzlich Übergangsbereiche von einem Bereich in einen anderen, von einem Draußen in ein Drinnen oder umgekehrt. In ihrer Gestaltung spiegeln sich die soziale, funktionale und symbolische Bedeutung eines Gebäudes und dessen Verantwortlicher wider. Der Südwesteingang des Palastes von Knossos war vermutlich einer der Haupteingänge in der endpalastzeitlichen Nutzungsphase. Er stellte die Schnittstelle zwischen dem Westhof und dem >Prozessionskorridor< dar, der von dort in das Innere des Palastes, möglicherweise bis zum Zentralhof, führte (Abb. 1. 2).

Den Eingangsbereich erreichte man über einen der erhöhten Platten- oder >Prozessionswege<, die von der im Westen gelegenen Stadt zum Westhof führten und sich dort mehrmals verzweigten, um in verschiedene Bereiche und Eingänge des Palastareals zu münden. Der zum Eingang führende Plattenweg verlief entlang der Westfassade des Palastes, vorbei an zwei Altären und traf vor der im Süden des Westhofs gelegenen Eingangsportikus rechtwinklig auf einen von Westen nach Osten verlaufenden Plattenweg. Letzterer gehörte bereits $\mathrm{zu}$ einer früheren Eingangssituation und bildete nun gleichzeitig eine Art Schwelle zwischen Westhof und Eingangsportikus. Jenseits dieses quer gelagerten Plattenweges setzte sich der Weg fort, um nach etwa drei Metern erneut auf einen quer gelagerten Plattenweg zu treffen, der eine zu rekonstruierende Tür in der Westwand der Eingangsportikus mit einem weiter östlich entlang der Palastfassade verlaufenden Plattenweg verband. Auf dessen Fortset- 
zung nach Süden gelangte man durch eine Tür in den >Prozessionskorridor . Parallel zu letztgenanntem Plattenweg führte ein weiterer Weg in den von Arthur Evans als »Reception Area bezeichneten, im Westen an den Prozessionskorridor angrenzenden Raum9.

Die Bodenflächen zwischen den Plattenwegen waren gepflastert und rot verputzt $t^{10}$. Die Wände der Eingangsportikus waren mit Malereien versehen, von denen sich ringsum eine Steinplatten imitierende, ursprünglich ca. $40 \mathrm{~cm}$ hohe Zierleiste erhalten hat. Darüber war zumindest an der Ostwand der Eingangsportikus ein Stier dargestellt, wie sich am in situ dokumentierten Rest der Darstellung eines Vorderbeins erkennen lässt ${ }^{11}$. Der etwa in Lebensgröße wiedergegebene Stier war aus dem Palast heraus und in Richtung des Westhofs orientiert. Aufgrund des fragmentarischen Erhaltungszustandes muss offenbleiben, ob es sich allein um einen nach außen stürmenden Stier oder, wie von Evans vermutet, um eine der typischen Stiersprungszenen handelte ${ }^{12}$.

Welche Funktion die Darstellung des Stieres oder Stiersprungs in dem Eingangsbereich ausgeübt haben könnte, wurde bereits mehrfach diskutiert. Nannò Marinatos zufolge hatte der Stier in Eingangsräumen keine Wächterfunktion. Er war vielmehr das stärkste Tier, die gewaltigste Kraft der Natur, die das bronzezeitliche Kreta kannte, und hätte in den Eingangsräumen somit in erster Linie als ein Emblem der Macht fungiert ${ }^{13}$. Nach Birgitta und Erik Hallager waren Stier und Stiersprung Motive, welche sich auch aufgrund ihres Vorkommens in anderen Bildmedien eng mit dem Palast von Knossos bzw. mit den darin agierenden Personen verknüpfen ließen. Der Stier in den Eingangsräumen hätte daher vor allem dazu gedient, die »Macht des Herrschers zu symbolisieren « ${ }^{14}$. Marika Zeimbekis hingegen stellte derartige Deutungen angesichts des sonstigen Fehlens von Herrscherikonographie in Frage. Sie wies darauf hin, dass in Knossos durch einige der Wandbilder eine Art dauernder >Vergegenwärtigung < des obersten Amtsinhabers suggeriert würde ${ }^{15}$. Die Platzierung von Stierbildern in den Eingangsbereichen wäre daher vielmehr als ein »kosmologisches Statement« zu verstehen und hätte, wie in Ägypten und Mesopotamien, die von den Machtinhabern ausgeübte Kontrolle zerstörerischer Kräfte zum Ausdruck gebracht ${ }^{16}$.

Wie sich anhand unterschiedlicher Bildgattungen erschließen lässt, reicht die Tradition der Stierthematik in der minoischen Kultur bis an die Anfänge der Besiedlung Kretas zurück $^{17}$. Bereits aus der Vorpalastzeit sind Darstellungen von Stieren sowie von Stierfang und damit der Überwindung dieses mächtigen Tieres durch vorzugsweise männliche Mitglieder

Evans 1928, 673 Abb. 427.

Evans 1928,670 .

Evans 1928, 675 Abb. 428; Boulotis 1987, 148 Abb. 3.

Evans 1928, 677 Abb. 429. Zu den Resten weiterer Stiermotive in Eingangsbereichen s. Blakolmer 2001,

32; Hallager - Hallager 1995, 547-548; Marinatos 1989, 26; Marinatos 1996, 150-151; Shaw 1995, 97-98 Abb. 8.

13 Marinatos 1996, 151.

14 Hallager - Hallager 1995, 549.

15 Zeimbekis 2006, 33.

16 Zeimbekis 2006,33 .

17 Zeimbekis 2006, 28-37. 
der Gesellschaft bekannt ${ }^{18}$. Zeimbekis zufolge organisierte und formalisierte die knossische Elite Rituale der Unterwerfung und Beherrschung des Stiers, welche bereits lange vor der Etablierung der Palastgesellschaft existiert hatten ${ }^{19}$. In seiner sakrobatischen Form wurde das Überwinden des Stieres nun möglicherweise von den jungen Männern der knossischen Oberschicht praktiziert, die dadurch, so Marinatos, ihre Fähigkeit, das mächtigste Tier Kretas zu beherrschen und zu kontrollieren, zum Ausdruck brachten ${ }^{20}$. Die Motive des Stieres und vor allem des Stiersprungs, die nicht nur symbolisch auf tief in der minoischen Kultur verankerte Ideen und Rituale verwiesen, sondern spätestens seit der Neupalastzeit auch von der knossischen Palastelite zur Demonstration ihrer Skills für sich beansprucht wurden, stellten somit wohl die eindrucksvollsten Bilder dar, um den Palastbesucher mit den Ideen und Vorstellungen zu konfrontieren, die das Palastgeschehen und insbesondere dessen Verantwortliche charakterisierten.

Das Muster von erhöhten Plattenwegen, welches sich deutlich vom umgebenden roten Verputz abzeichnet, erlaubt in diesem Zusammenhang weitere Überlegungen hinsichtlich des Bewegungsmusters, welchem der vielleicht in einer Prozession durch den Eingangsbereich Schreitende gefolgt sein könnte. Auf dem Weg, der über den Westhof führte, erblickte man zunächst die zentrale Säule, im weiteren die oberhalb des Steinimitats ursprünglich vermutlich ebenfalls mit einem Wandbild dekorierte Portikusrückwand sowie den Eingang in die Reception Area. Man überquerte den ersten, in West-Ost-Richtung kreuzenden Plattenweg und umrundete dann auf der Fortsetzung die zentral platzierte Säule, indem man auf dem nächsten quer gelagerten Plattenweg rechtwinklig nach links umbog. Erst auf diesem Plattenweg bot sich das Stier[sprung]motiv an der Wand in seiner vollen Ausdehnung dar. Die lebensgroße Darstellung des Stieres sowie eventuell zugehöriger Stierspringer, auf die man sich nun auf einer Strecke von knapp sieben Metern zu bewegte, dürfte ein imposantes Moment geschaffen haben, in dem sich dem Eintretenden die Bedeutung des Palastes bzw. der darin herrschenden Elite, die den Stiersprung als ihr Emblem zeigte, offenbarte. Kurz vor der Wand bog man auf dem nächsten Plattenweg rechtwinklig nach rechts ab, um an dem eindrucksvollen Bild entlang zur eigentlichen Eingangstür in den >Prozessionskorridor $\mathrm{zu}$ schreiten.

Der so beschriebene Pfad stellt eine der Möglichkeiten dar, unter Einhaltung des von den Plattenwegen vorgegebenen Bewegungsschemas vom Westhof zur Eingangstür in den >Prozessionskorridor $\mathrm{zu}$ gelangen ${ }^{21}$. Die Annäherung an das Palastinnere wäre demzufolge von mehrmaligen Richtungswechseln begleitet gewesen, die möglicherweise die hintereinander erfolgende Wahrnehmung unterschiedlicher visueller Eindrücke und somit die

18 s. auch Sikla 2003, 377-379. 383 Taf. 3. Auch Übergangsriten in den Erwachsenenstatus werden in diesem Zusammenhang oftmals genannt, s. Panagiotopoulos 2006a, 126 Anm. 5 mit Literaturangaben.

19 Zeimbekis 2006, 34 .

20 Marinatos 1994, 93.

21 Eine Alternative wäre das Umbiegen nach links bereits auf dem ersten quer gelagerten, von Westen kommenden Plattenweg, der in einer früheren Phase des Palastes die direkte Verbindung zwischen Westhof und 
stückweise Erfahrung des Eintretens in das Gebäude zum Ziel hatten. In diesem Sinne erweist sich der Eingangsbereich in den Palast von Knossos als ein Bild-Raum, der in seiner Anlage auf die srichtige < räumliche Annäherung und sukzessive Sinnkonstruktion abzielte. Er war geprägt von einem der urtümlichsten Motive der kretischen Kultur und dafür konzipiert, dem Herannahenden eindrucksvoll die Bedeutung der Palastinhaber zu vermitteln und diesen auf das vor ihm liegende Geschehen einzustimmen. Erst nachdem er selbst den Stier im Vorbeigehen süberwunden< hatte, betrat er durch eine Doppeltür den langen, dunklen >Prozessionskorridor<.

\subsection{Der >Prozessionskorridor im Palast von Knossos}

Im >Prozessionskorridor setzte sich der erhöhte, weiße Plattenweg zwischen rot verputzten Bodenflächen fort (Abb. 1-3). Der 3,3 m breite Gang führte zunächst nach Süden, bog Evans zufolge nach etwa $24 \mathrm{~m}$ nach links bzw. nach Osten um, führte im Geschoss über den South Terrace Basements etwa $48 \mathrm{~m}$ lang in östliche Richtung, bog dann noch einmal nach links bzw. Norden um und mündete schließlich nach weiteren $22 \mathrm{~m}$ in den Zentralhof ${ }^{22}$. Der >Prozessionskorridor $<$ bildete damit einen Abschnitt der wichtigen Verbindungsroute zwischen der Stadt, dem Westhof als Schnittstelle zwischen Stadt und Palast sowie dem Zentralhof als Zielort des Wegesystems ${ }^{23}$.

Die lang gestreckte architektonische Form des Korridors sowie die Fortsetzung des zentral verlaufenden, erhöhten Plattenwegs legen nahe, dass es sich hier in erster Linie um einen Durchgangsraum handelte. Die Anlage des Weges innerhalb der Gebäudestruktur macht zudem deutlich, dass diese einer künstlichen Verlängerung der Verbindung zwischen dem Westhof und dem Zentralhof diente ${ }^{24}$. Möglicherweise sollte hiermit eine Intensivierung der Erfahrung des Eintritts in den Palast oder des Übergangs von dem einer größeren Gruppe von Menschen zugänglichen Westhof in den exklusiveren Bereich des Zentralhofs erreicht werden. Der Akt des Durchschreitens des Korridors spiegelte sich auch in den Bildern an den Wänden wider.

Von den Darstellungen auf beiden Seiten des Korridors haben sich die Füße sowie in Einzelfällen die Körper der Figuren erhalten. Orientierung, Gewänder sowie die kon-

Zentralhof darstellte. Da jedoch, wie in Evans 1928, 662 Abb. 423 zu sehen, die Plinthe der späteren Westfassade den Plattenweg teilweise überlagert, ist m. E. davon auszugehen, dass dieser in der besprochenen Nutzungsphase des Eingangsbereichs nicht mehr den primär genutzten Eingangsweg darstellte. Ausgeschlossen werden kann dies freilich nicht, da der entlang der dekorierten Wand verlaufende Plattenweg von jenem älteren Weg abzweigend bis in den >Prozessionskorridor< hineinführte.

22 Vgl. Evans 1935, Plan C.

23 Panagiotopoulos 2006b, 35-36 mit weiterer Literatur.

$24 \mathrm{Zu}$ den Umbaumaßnahmen, im Zuge derer die ursprünglich direkte Verbindung zwischen Westhof und Zentralhof zugebaut und der gleich verlaufende Vorgänger des >Prozessionskorridors < angelegt wurde, s. Evans $1928,660-670$. 

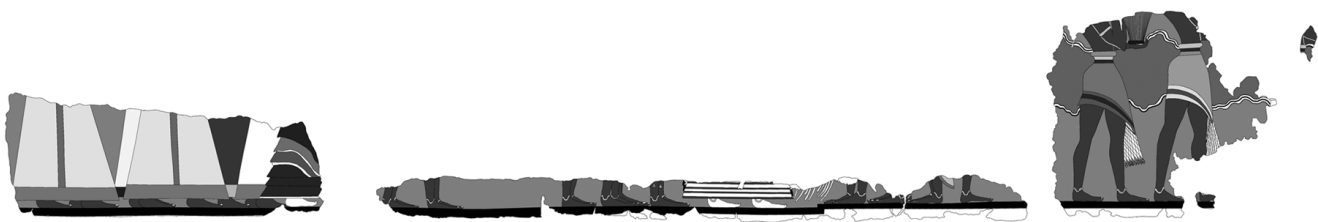

Abb. $3 \mid>$ Prozessionskorridor<, Fragmente der Darstellung an der Ostwand.

ventionelle rotbraune und weiße Farbgebung der Hautpartien zeigen, dass es sich um mehrere Gruppen annähernd lebensgroßer, männlicher und weiblicher Figuren handelte, die - entsprechend der Anlage des Korridors - vom Westhof in das Gebäudeinnere schritten $^{25}$ (vgl. Abb. 3). Lediglich eine Gruppe von vier männlichen Personen, von denen mindestens eine, wie Christos Boulotis nahe legte ${ }^{26}$, mit einem $>$ Fellrock $<$ bekleidet ist, war in die Gegenrichtung gewandt. Diese Gruppe steht zudem, dies wurde bisher in der Forschung vernachlässigt, hinter einer kleinen Stufe, und damit auf höherem Niveau als die vom Eingang herannahenden Prozessierenden. Wie andernorts ausführlich zu zeigen sein wird, dürfte es sich bei dieser Gruppe um Vertreter des Palastpersonals handeln ${ }^{27}$. Aufgrund ihrer Platzierung innerhalb der Bildkomposition lassen sie sich als bereits im Palast anwesend und den Hereinkommenden entgegen gewandt begreifen. Zu ihren Aufgaben gehörte es vielleicht, herbeigebrachte Gaben wie beispielsweise ein ebenfalls von Boulotis identifiziertes Textil ${ }^{28}$ in Empfang zu nehmen.

Der in Form dreier durch Wellenbänder voneinander getrennter Farbflächen gestaltete Hintergrund des Prozessionsgeschehens könnte, wie bereits von Wolfgang Schiering vermutet, eine in Malerei übersetzte Gipssteinvertäfelung darstellen ${ }^{29}$. In einer früheren Ausstattungsphase des Korridors existierte tatsächlich eine Gipssteinvertäfelung in diesem Bereich ${ }^{30}$, die im Zuge der Verbreiterung und anschließenden Innenraumgestaltung des Korridors nun in Malerei umgesetzt wurde. Sie legt nahe, dass diese bereits in der früheren Nutzungsphase vorhandene Form der Wandgestaltung auch im letzten >Prozessionskorridor als angemessener Innenraumdekor gewählt und imitiert wurde. Durch die auf den früheren Innenraumdekor rekurrierende Hintergrundgestaltung wurde folglich auch der spätere, bildlich vergegenwärtigte Prozessionszug in einen ähnlich konzipierten Durchgangsraum eingebettet.

$25 \mathrm{Zu}$ den spärlichen Fragmenten der Figurengruppen auf der gegenüberliegenden Westwand s. Boulotis 1987, 150 Abb. 5; Cameron 1987, 323 Abb. 4. 6.

26 Boulotis $1987,147$.

27 Eine ausführliche Darlegung der Neudeutung des >Prozessionsfreskos < befindet sich in Vorbereitung durch die Verf.; s. außerdem Günkel-Maschek 2011, 132-134; Günkel-Maschek im Druck.

28 Boulotis $1987,150.154$ Abb. 8.

29 Schiering 1960, 26-33. s. im Unterschied dazu die Deutung des Wellenbanddekors als Anspielung auf landschaftliche Konturen bei Evans 1928, 728; Blakolmer 2010, 97.

30 Evans 1928, 668 Abb. 425 . 
Die Übereinstimmung zwischen den Darstellungen an den Wänden und dem durch die architektonische Form und signifikante Bodengestaltung suggerierten Handlungsgeschehen im Korridor legen nahe, dass an den Wänden das zu bestimmten Zeiten tatsächlich hier stattfindende Geschehen reflektiert und verstetigt wurde. Die Lebensgröße der Figuren trug ihren Teil dazu bei, um in dem lang gestreckten und vermutlich recht dunkleKorridor die Hereintretenden in das Prozessionsgeschehen zu involvieren. Letzteres vermittelte ihnen vielleicht zusätzlich ein Gefühl des kollektiven Handelns, welches einen häufig begegnenden Aspekt minoischen Ritualgeschehens darstellte. Die mehrheitlich eingehaltene Bewegungsrichtung der Figurengruppen in das Gebäudeinnere verlieh dem Raum dabei einen starken richtungsweisenden Charakter ${ }^{31}$, der den Bewegungsfluss hin zum Zielort potenzierte. Gleichzeitig hielten die Darstellungen fest, aus welchen durch Gewänder und Gedrängtheit der Figuren differenzierten Gruppen sich der Menschenzug zusammensetzte und welche Gaben von wem in den Palast gebracht wurden. Nicht zuletzt veranschaulichten sie, wie die Figuren, die als Mitglieder des Palastpersonals gedeutet werden können (s. o.), im Rahmen des Prozessionsgeschehens die einzelnen Gruppen sowie ihre Gaben in Empfang nahmen ${ }^{32}$. Die Darstellungen im >Prozessionskorridor lassen sich somit als eine Verstetigung des Ritualgeschehens begreifen, in dem sich die soziale Gliederung der palastnahen Elite sowie die Bedeutung des Palastes selbst als Menschen und Gaben empfangender Instanz manifestierte. Die künstliche Verlängerung des Verbindungswegs zwischen Westhof und Zentralhof, auf dem wohl zu bestimmten Anlässen ähnliche Prozessionen in den Palast zogen, diente auf diese Weise nicht nur der Prolongierung und Intensivierung des Aktes des Einzugs in den Palast, sondern zugleich der visuellen Kommunikation von Sachverhalten, die das Geschehen und die Ordnung im Palast konstituierten.

\subsection{Der Thronraum im Palast von Knossos}

In Hinblick auf seine Langlebigkeit, die erhaltene Ausstattung sowie die bildliche Wandgestaltung stellte der Thronraum vermutlich einen der wichtigsten Orte des Geschehens im Palast von Knossos dar (Abb. 1. 4). Vom Zentralhof, dessen Westfassade Eingänge in diverse Raumkomplexe bot, gelangte man zunächst durch eine viertürige pier-and-door partition und über zwei, später vier ${ }^{33}$ Stufen nach unten in den Vorraum des Thronraums. Entlang der Wände zu beiden Seiten waren niedrige Steinbänke aufgestellt. Die Wandmalereien oberhalb dieser Bänke zeigten zunächst eine Leiste aus Steinimitat, über der sich,

31 s. dazu auch Cameron 1970, 165; Hägg 1985, 210-211.

32 Das von Evans im südlichen Bereich des Palastes geborgene und zum >Prozessionskorridor< gehörende Fragment eines weiteren >Fellrock<-Trägers, welches von Christos Boulotis (1987, 149 Abb. 4 a; 4 b) vorgelegt und ergänzt wurde, deutet auf die Beteiligung weiterer >Fellrock<-Träger am Prozessionsgeschehen hin.

33 Erst mit der zweiten Erhöhung des Zentralhofniveaus in SM IIIA1 wurden die oberen zwei Stufen angelegt, vgl. Mirié 1979, 56-57 Taf. 10; Niemeier 1986, 68. 93. 
zumindest auf der links gelegenen Südwand, ein Stier erhob, der auf den Eintretenden zu schritt $^{34}$. Hinter einer weiteren, zweifachen Türöffnung ${ }^{35}$ lag schließlich der Thronraum selbst.

Hier befanden sich ein >Lustralbecken`zur Linken und im südlichen Bereich eine Tür in der dem Vorraum gegenüberliegenden Westwand, der steinerne Thron an der Nordwand zur Rechten sowie weitere niedrige Bänke, die an Nord- und Westwand wie auch an der Einfassungsmauer des >Lustralbeckens entlang verliefen. Die Tür in der Westwand führte zunächst in einen von Evans als »Inneres Heiligtum« bezeichneten Raum mit Podest gegenüber der Tür und von dort in die nördlich gelegene Raumfolge der Service Section. Über eine weitere Tür hinter dem Lustralbecken in der Südwestecke des Thronraums gelangte man in südlich angrenzende Magazinräume.

Wie im Vorraum war auch im Thronraum der Boden gepflastert, wobei hier zusätzlich eine rot bemalte Verputzschicht aufgetragen worden war ${ }^{36}$. Rot war auch die dominante Farbe an den Wänden. So waren die Wände des >Lustralbeckens く bis auf eine Zierleiste im oberen Wandbereich vollständig rot bemalt. Auf der Nord- und Westwand des Thronraums bestand die Hintergrundgestaltung aus vier horizontalen, alternierend roten und elfenbeinfarbenen Flächen mit Wellenkontur, vor denen vereinzelt platzierte Papyrus-Schilfpflanzen sowie Palmen zu beiden Seiten des Throns eine idealisierte Flusslandschaft suggerierten ${ }^{37}$. In dieser Landschaft befanden sich die lagernden Greifen, die sowohl den Thron als auch die Tür in der Westwand flankierten. Nach unten zu den Bänken hin schloss die Wandmalerei mit einer Leiste aus Steinimitat ab. Stilisierte bikonkave Basen bzw. Friese aus aneinander grenzenden, konvexen Elementen waren an den Wänden zwischen Bänken und Thron platziert. Nach oben hin, in der Fläche oberhalb des Hauptbildfeldes der Wände schlossen schließlich noch zwei, lediglich durch Zierleisten getrennte Register rot bemalter Flächen an. Das insgesamt von der Farbe Rot dominierte Erscheinungsbild des Thronraums, welches Parallelen in neupalastzeitlichen Kulträumen wie dem >Lustralbecken < im Palast von Kato Zakros findet ${ }^{38}$, dürfte dem Raum eine sinnlich wie symbolisch bedeutsame Atmosphäre verliehen haben, die den angemessenen Rahmen für das Geschehen im Umkreis des Throns darstellte.

Bereits Wolf-Dietrich Niemeier fasste die konstitutiven Elemente Thron und Throninhaber sowie Palmen, Greifen und ornamental-symbolische Friese zu beiden Seiten zu einem

34 Evans 1935, 893 Abb. 872. Wieder ist nur ein Huf, diesmal ein Hinterhuf, erhalten, weswegen nicht ausgeschlossen werden kann, dass es sich auch in diesem Fall um eine Stiersprungszene handelte. Die Position des Fußes legt jedoch nahe, dass der Stier nicht im stürmischen Lauf dargestellt war, wie dies für Stiersprungszenen typisch ist.

35 Vgl. Niemeier 1986, 93-94, wonach in der vierten und letzten Nutzungsphase des Thronraums, die Niemeier der mykenischen Okkupation zuordnet, der zentrale Pfeiler entfernt wurde, um ein Megaron zu erschaffen.

36 Evans 1935, 902-903 Abb. 877.

37 Niemeier 1986, 88; Evans 1935, 908. 910.

38 Platon $1971,183$. 


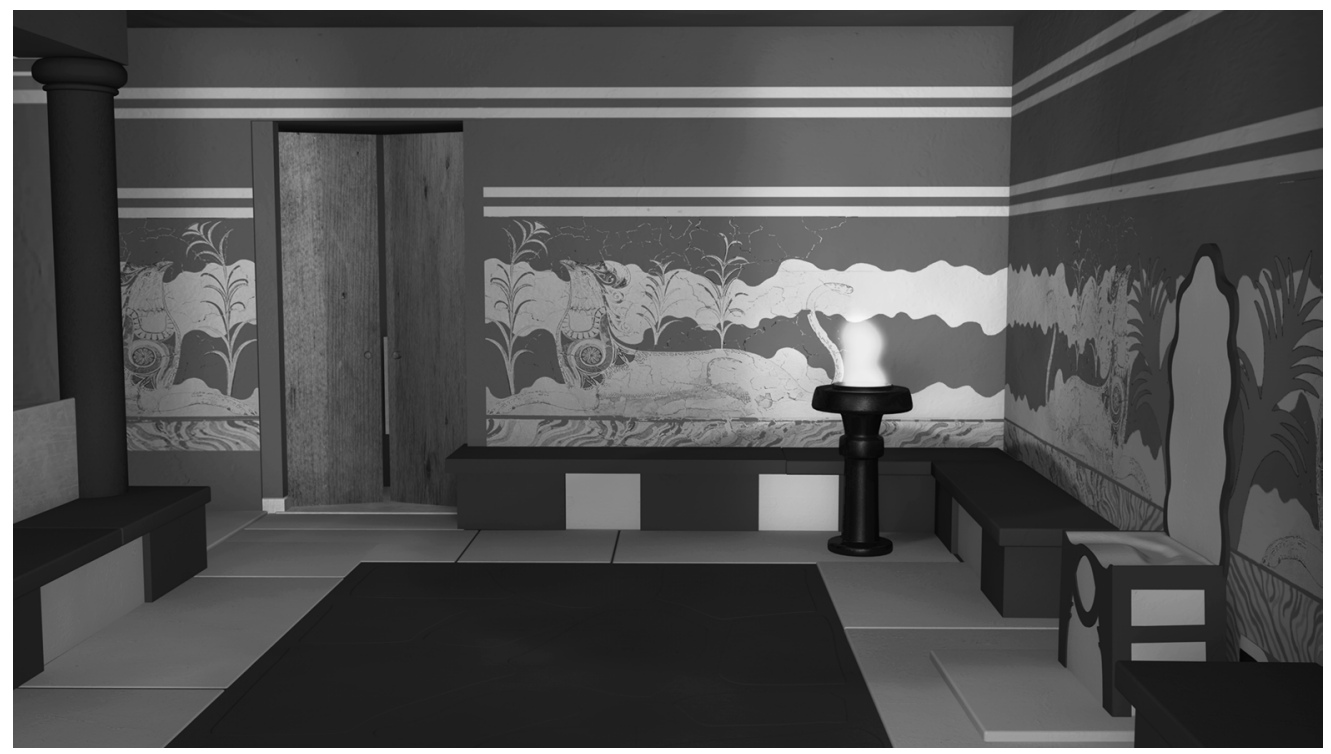

Abb. 4a | Thronraum, Blick vom Vorraum aus.

»Gottheit-Ideogramm« zusammen ${ }^{39}$. Ihm zufolge trugen die gemalten Elemente dazu bei, die Göttlichkeit der thronenden Person zu unterstreichen bzw. visuell zu veranschaulichen. In ähnlicher Weise fungierten die Greifen zu beiden Seiten der Tür in der Westwand, die eine Person flankierten, welche durch diese Tür in den Thronraum trat.

Die zu beiden Seiten des Throns und der Tür in der Westwand platzierten Greifen (Abb. 4 a. b), die sich einerseits in der artifiziellen Flusslandschaft befinden, andererseits explizit auf architektonische Elemente im tatsächlichen Thronraum Bezug nehmen, stellen eine Verknüpfung zwischen der künstlich geschaffenen Umgebung und dem realen, architektonisch gefassten Raum bzw. einzelnen darin an bestimmten Stellen agierenden Personen her und suggerieren den übernatürlichen Landschaftsraum als Rahmen und virtuellen Ort des im Thronraum stattfindenden Handlungsgeschehens. Eine aus dem >Inneren Heiligtum < in den Thronraum tretende Person wurde vom Vorraum aus als frontal und von Greifen flankiert wahrgenommen, ein bild-räumliches Arrangement, welches bereits mehrmals mit Siegeldarstellungen in Zusammenhang gebracht wurde, in denen Greifen zu beiden Seiten einer weiblichen Figur auf erhöhten Grundlinien stehen. Dieses Motiv könnte die an den Wänden oberhalb der Bänke platzierten Greifen zu beiden Seiten der Tür in der Westwand reflektieren ${ }^{40}$. Sollte es sich bei dieser Gruppe von Siegelbildern tatsächlich um Anspielungen auf den knossischen Thronraum handeln, so ließen sich 


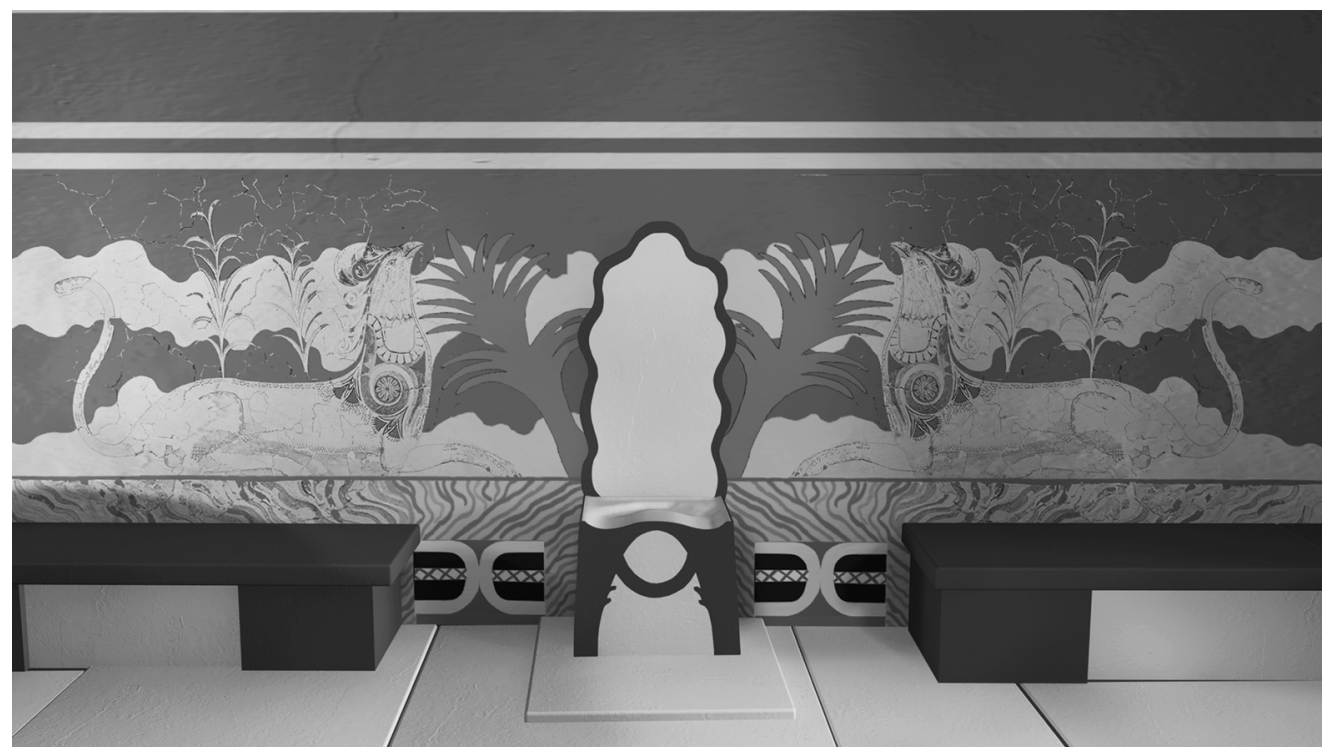

Abb. 4b | Thronraum, Frontaler Blick auf den Thron.

diese Darstellungen also möglicherweise auf die zwischen den Greifen >erscheinende Person in der Westtür beziehen ${ }^{41}$. Bild und Moment der >Erscheinung < einer von Greifen flankierten weiblichen Person entsprachen demnach möglicherweise einem in der religiösen Vorstellung verankerten Ensemble, das im Thronraum durch die artifizielle Hinzugesellung der Greifen an den Wänden in seiner Vollständigkeit realisiert wurde.

Ähnliche Überlegungen lassen sich für das bildräumliche Thronensemble aufstellen. Die Platzierung des Throns an der Nordwand und damit an der vom Vorraum aus gesehen rechten Seite stellt ein sehr eigentümliches räumliches Arrangement dar, aus dem sich jedoch, unter Einbeziehung der bildlichen Wandgestaltung zu beiden Seiten des Throns, eine spezifische Abfolge des >Sehens der thronenden Person rekonstruieren lässt. Näherte man sich vom Zentralhof kommend durch den Vorraum, so wurde der oder die Thronende zunächst im Profil wahrgenommen (Abb. 4 a). Erst wenn man vor dem Thron stand und sich frontal dem Throninhaber zuwandte, konnte das Thronarrangement einschließlich der bildlich vergegenwärtigten Greifen, Palmen und bikonkaven Basen zu beiden Seiten des Throns vollständig wahrgenommen werden (Abb. 4 b). Die Platzierung des Throns an der rechten Wand einschließlich der Platzierung der Wandbilder diente daher möglicherweise der Inszenierung eines ganz bestimmten Erscheinungsbildes bzw. eines ganz bestimmten Ablaufs der sukzessiven Wahrnehmung der thronenden Person. Das 
>Lustralbecken als weitere Installation im Thronraum könnte in diesem Zusammenhang als Standort für den frontalen Blick auf die Person auf dem Thron gedient haben - falls es nicht ausschließlich letzterer vorbehalten war ${ }^{42}$.

Wie die in der westlichen Tür serscheinende< Person, so könnte auch das vom Vorraum aus wahrgenommene, räumliche Arrangement der thronenden Person in Siegelbildkompositionen reflektiert worden sein. In mehreren Abdrücken ist auf stets im rechten Bildfeld platzierten Sitzen oder als Sitz dienenden architektonischen Strukturen eine thronende weibliche Figur dargestellt ${ }^{43}$. Ihr nähern sich Figuren von links, deren Handlungen Zuwendungen an bzw. Interaktionen mit der verehrten Gottheit wiedergeben. Greifen fehlen in diesen Darstellungen - sie wären auch in Realität kaum neben der im Profil wahrgenommenen, thronenden Person zu sehen gewesen.

Das architekturräumliche Arrangement einschließlich der bildlich vergegenwärtigten Elemente legt folglich nahe, dass je nach Anlass des Geschehens und je nach Grad des Vordringens des Betrachters in Vorraum oder Thronraum das Erscheinungsbild der im Thronraum lokalisierten und von Greifen begleiteten Person variierte. Die Siegelbilder geben möglicherweise eine Vorstellung davon, wie jene Sichtweisen in Bildform festgehalten wurden und welche Ideen oder Aktionen darüber hinaus mit der jeweiligen Erscheinungsform der Person assoziiert wurden. Sind sie außerdem tatsächlich, wie mehrfach vermutet wurde, auf das Geschehen im Thronraum von Knossos zu beziehen, so offenbart sich einmal mehr die Bedeutung, die diesem (Handlungs-)Raum in der minoischen Bild- und Lebenswelt beigemessen wurde.

Gerade diese Bedeutung war wohl auch der Grund, weshalb der Thronraum während der Jahrhunderte seines Bestehens immer wieder an neue Verhältnisse angepasst wurde. So wurde in der späten Neupalastzeit das >Lustralbecken zugeschüttet: Dies bedeutete das Ende der ursprünglich darin durchgeführten Rituale. Mit dem Ende der Endpalastzeit wurde die Tür, die von der im Norden gelegenen Service Section in das >Innere Heiligtum< führte, blockiert ${ }^{44}$, so dass auch ein >Erscheinen< durch diese Tür nicht mehr in der bisherigen Form durchgeführt werden konnte. Was jedoch bis zum Ende des Palastes zumindest dem Erscheinungsbild nach unverändert blieb, war das bild-räumliche Arrangement des Throns. Mit der Aufgabe oder Veränderung einiger Praktiken im Thronraum könnte sich auch die Funktion des Throninhabers gewandelt haben. Was der Hintergrund dieser Veränderungen war, und wer in den Jahrzehnten der Endpalastzeit bis zur mykenischen Übernahme um 1375 v. Chr. ${ }^{45}$ auf dem Thron saß, darüber lassen sich nur Vermutungen anstellen. In der mykenischen Zeit schließlich dürfte man den Thronraum aufgrund

42 Es kann nicht ausgeschlossen werden, dass die Person, die in der Westtür serschien $<$ und auf dem Thron Platz nahm, auch das >Lustralbecken< nutzte. Möglicherweise geschah die rituelle Nutzung von Thron, Westtür und >Lustralbecken< in Abhängigkeit von bestimmten Zeitpunkten im Jahr, vgl. Goodison 2004, 342-346.

43 z. B. CMS II.7, 8; II.8, 268; V, 199; I, 101; XI, 30.

44 Niemeier 1986, 93.

45 Nach Niemeier 1986, 93-94. Vgl. auch Momigliano 2007, 7 Taf. o, 2. 
seiner zentralen Position als Sitz für den neuen Herrscher gewählt haben ${ }^{6}$. Nach Niemeier sprechen bauliche Veränderungen in dieser Zeit dafür, dass der Raumkomplex aus Vorraum und Thronraum nun in ein Megaron abgeändert wurde. Der neue Repräsentativraum sollte bald das Vorbild der Megara der mykenischen Paläste bilden, die sowohl die seitliche Platzierung des Throns als auch die Bildkomposition für dessen Rahmung übernahmen ${ }^{47}$. Die Langlebigkeit des Thronarrangements - trotz der Transformationen des mit ihm assoziierten Handlungsraums - deutet darauf hin, dass die Begleitung durch Greifen zu jeder Zeit geeignet erschien, die herausragende Bedeutung und übernatürliche Macht der im Herzen des Palastes thronenden Person eindrucksvoll zu vergegenwärtigen.

\section{Zusammenfassung}

Das Konzept des Bild-Raums, in dem die Rolle von Bildwerken als prägenden Elementen von (Handlungs-)Räumen hervorgehoben wird, eröffnet neue Perspektiven auf den Einsatz von Bildern in der minoischen Kultur. Am Beispiel der Wandbilder wurde dargelegt, wie diese als Bestandteile ihrer räumlichen Kontexte begriffen werden können, wobei jene räumlichen Kontexte nicht nur materiell-architektonische Strukturen bezeichnen, sondern auch das mit ihnen in Zusammenhang stehende Handeln einbeziehen. In diesem Sinne wurden die (Handlungs-)Räume aufgrund ihrer Lage und Form in Eingangsbereiche, Durchgangsräume und >Aufenthaltsräume< differenziert. Die Wandbilder wiesen dementsprechend unterschiedliche Motive und Kompositionen auf, die in Hinblick auf Wahrnehmung und Handeln angebracht waren. Sie veranschaulichten die mit den Funktionen der einzelnen Bereiche assoziierten Ideen und Vorstellungen und verliehen so jedem (Handlungs-)Raum eine angemessene thematische oder symbolische Konnotation.

Im Südwesteingang wurden die auf den >Prozessionswegen sonen mit dem Motiv des Stiers oder Stiersprungs konfrontiert, welches die damit verknüpften und tief in der minoischen Kultur verankerten Vorstellungen als für den Palast von Knossos repräsentative Thematik vor Augen führte. Im anschließenden >Prozessionskorridor fand sich der Hereinkommende in einer Masse von Menschen wieder, die sich wie er durch den langen Gang dem Zentralhof näherten. Dem Prozessionsgeschehen entgegen gewandt dargestellte Mitglieder des Palastpersonals hielten dabei möglicherweise fest, wer in letzter Instanz als Empfänger der realen und gemalten Menschengruppen und Gaben hervortrat. Im Thronraum schließlich, dem langlebigsten Bild-Raum des minoischen Kreta, traf man auf eine von Greifen begleitete Person, deren herausragende

46 Pelon 1983, 255; Niemeier 1986, 93-95.

47 Vgl. dazu auch den Beitrag von Ulrich Thaler in diesem Band. 
Stellung in Palast und Gesellschaft mittels des bedeutungsvollen und sich je nach dem Standort des Betrachters unterschiedlich darstellenden Thronarrangements effektvoll zum Ausdruck gebracht wurde.

Die Wandbilder im Palast von Knossos können damit als ein wesentliches Instrument erkannt werden, mittels dessen das in bestimmten Räumlichkeiten stattfindende Geschehen genau die Sinnkontexte erhielt, welche in der minoischen Vorstellungswelt mit den beteiligten Personen und vollzogenen Handlungen assoziiert wurden. Sie bedienten sich dabei eines motivischen Vokabulars, welches auch sonst in der reich bebilderten Lebenswelt der palastnahen Elite allgegenwärtig war. So, wie sie appliziert auf Gebrauchsobjekten oder in plastischer Form in der Siegelpraxis, der Kultpraxis und anderen Aktivitäten relevante Ideen und Vorstellungen vergegenwärtigten, waren die Bilder auch als prägende Elemente bestimmter architekturräumlicher Kontexte und der Handlungen, für die letztere angelegt waren, unentbehrlich. Mit den unterschiedlichen Formen von durch Wanddekor charakterisierten Bild-Räumen lassen sich folglich Aspekte der Konstitution von Sinngebilden erfassen, in denen die Verknüpfungen zwischen Ideen und Praktiken an konkreten Orten zu Tage treten. Als symbolisch konnotierte Orte bestanden die bebilderten Eingänge, Durchgangsbereiche und >Aufenthaltsräumeく nicht zuletzt auch über das aktuelle Handlungsgeschehen hinaus, so dass hier dauerhaft die Ideen und Vorstellungen präsent waren, die in ihrer einstigen Gesamtheit die Bedeutung und Funktion des minoischen Zentrums ausmachten.

\section{Bibliographie}

\section{Blakolmer 1995}

F. Blakolmer, Komparative Funktionsanalyse des malerischen Raumdekors in minoischen Palästen und Villen, in: R. Laffineur - W. D. Niemeier (Hgg.), Politeia. Society and State in the Aegean Bronze Age. Proceedings of the 5th International Aegean Conference, University of Heidelberg, Archäologisches Institut, 10-13 April 1994, Aegaeum 12 (Liège 1995) 463-474.

\section{Blakolmer 2000}

F. Blakolmer, The Functions of Wall Painting and Other Forms of Architectural Decoration in the Aegean Bronze Age, in: S. Sherratt (Hg.), The Wall Paintings of Thera. Proceedings of the First International Symposium, Petros M. Nomikos Conference Centre, Thera, Hellas, 30 August - 4 September 1997 (Athen 2000) 393-412. 


\section{Blakolmer 200I}

F. Blakolmer, Das minoisch-mykenische Stuckrelief. Zur Definition einer palatialen Kunstgattung der ägäischen Bronzezeit, in: F. Blakolmer - H. Szemethy (Hgg.), Akten des 8. Österreichischen Archäologentages am Institut für Klassische Archäologie der Universität Wien vom 23. bis 25. April 1999 (Wien 2001) 18-36.

\section{Blakolmer 2010}

F. Blakolmer, Small Is Beautiful. The Significance of Aegean Glyptic for the Study of Wall Paintings, Relief Frescoes, and Minor Relief Art, in: I. Pini - W. Müller (Hgg.), Die Bedeutung der minoischen und mykenischen Glyptik. VI. Internationales SiegelSymposium, Marburg, 9.-12. Oktober 2008, CMS Beih. 8 (Mainz 2010) 91-108.

\section{Boulotis 1987}

C. Boulotis, Nochmals zum Prozessionsfresko von Knossos: Palast und Darbringung von Prestige-Objekten, in: R. Hägg - N. Marinatos (Hgg.), The Function of the Minoan Palaces. Proceedings of the Fourth International Symposium at the Swedish Institute at Athens, 10-16 June 1984 (Stockholm 1987) 145-156.

\section{Cameron 1970}

M. A. S. Cameron, New Restorations of Minoan Frescoes from Knossos, BICS 17, 1970, 163-166.

\section{Cameron 1978}

M. A. S. Cameron, Theoretical Interrelations among Theran, Cretan and Mainland Frescoes, in: C. G. Doumas (Hg.), Thera and the Aegean World I. Papers Presented at the Second International Scientific Congress, Santorini, Greece, August 1978 (London 1978) $579-592$.

\section{Cameron 1987}

M. A. S. Cameron, The >Palatial< Thematic System in the Knossos Murals. Last Notes on Knossos Frescoes, in: R. Hägg - N. Marinatos (Hgg.), The Function of the Minoan Palaces. Proceedings of the Fourth International Symposium at the Swedish Institute at Athens, 10-16 June, 1984 (Stockholm 1987) 187-193.

\section{Evans 1928}

A. Evans, The Palace of Minos: a Comparative Account of the Successive Stages of the Early Cretan Civilization as Illustred by the Discoveries at Knossos II (London 1928).

\section{Evans 1935}

A. Evans, The Palace of Minos: a Comparative Account of the Successive Stages of the Early Cretan Civilization as Illustred by the Discoveries at Knossos IV (London 1935). 


\section{Goodison 2004}

L. Goodison, From Tholos Tomb to Throne Room: Some Considerations of Dawn Light and Directionality in Minoan Buildings, in: G. Cadogan - E. Hatzaki - A. Vasilakis (Hgg.), Knossos: Palace, City, State. Proceedings of the Conference in Herakleion Organised by the British School at Athens and the 23rd Ephoreia of Prehistoric and Classical Antiquities of Herakleion, in November 2000, for the Centenary of Sir Arthur Evans's Excavations at Knossos, BSA Studies 12 (London 2004) 339-350.

\section{Günkel-Maschek 20II}

U. Günkel-Maschek, Visual Basic? Überlegungen zur Korrelation von Ikonographie und Raumfunktion in der minoischen Architektur, in: F. Blakolmer - C. Reinholdt J. Weilhartner - G. Nightingale (Hgg.), Österreichische Forschungen zur ägäischen Bronzezeit 2009. Akten der Tagung am Fachbereich Altertumswissenschaften der Paris-Lodron-Universität Salzburg vom 6. bis 7. März 2009 (Wien 2011) 125-136.

\section{Günkel-Maschek im Druck}

U. Günkel-Maschek, Unterwegs im Labyrinth. Zur Gestaltung des >Prozessionskorridors< im spätminoischen >Palast< von Knossos, in: C. Reinholdt - W. Wohlmayr (Hgg.), Akten des 13. Österreichischen Archäologentages am Fachbereich Altertumswissenschaften der Paris-Lodron-Universität Salzburg vom 25. bis 27. Februar 2010 (im Druck).

\section{Hägg 1985}

R. Hägg, Pictorial Programmes in the Minoan Palaces and Villas?, in P. Darcque J.-C. Poursat (Hgg.), L'iconographie minoenne. Actes de la Table Ronde d'Athènes (21-22 avril 1983), BCH Suppl. 11 (Paris 1985) 209-217.

\section{Hallager - Hallager 1995}

B. Hallager - E. Hallager, The Knossian Bull - Political Propaganda in Neo-Palatial Crete?, in: R. Laffineur - W.-D. Niemeier (Hgg.), Politeia. Society and State in the Aegean Bronze Age. Proceedings of the $5^{\text {th }}$ International Aegean Conference, University of Heidelberg, Archäologisches Institut, 10-13 April 1994, Aegaeum 12 (Liège 1995) 547-555.

\section{Hood - Taylor I98I}

S. Hood - W. Taylor, The Bronze Age Palace at Knossos. Plans and Sections (London 1981).

\section{Löw 200I}

M. Löw, Raumsoziologie (Frankfurt a. M. 2001).

\section{Marinatos 1989}

N. Marinatos, The Bull as an Adversary: Some Observations on Bull-Hunting and Bull-

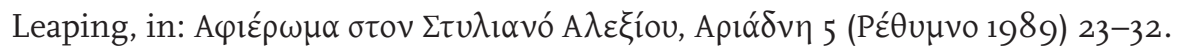




\section{Marinatos 1994}

N. Marinatos, The Export Significance of Minoan Bull-Leaping Scenes, Ägypten und Levante 4, 1994, 89-93.

\section{Marinatos 1996}

N. Marinatos, The Iconographical Program of the Palace of Knossos, in: M. Bietak (Hg.), Haus und Palast im Alten Ägypten (Wien 1996) 149-157.

\section{Mirié I979}

S. Mirié, Das Thronraumareal des Palastes von Knossos. Versuch einer Neuinterpretation seiner Entstehung und seiner Funktion (Bonn 1979).

\section{Momigliano 2007}

N. Momigliano, Introduction, in: N. Momigliano (Hg.), Knossos Pottery Handbook. Neolithic and Bronze Age (Minoan), BSA Studies 14 (London 2007) 1-8.

\section{Niemeier 1983}

W.-D. Niemeier, The Character of the Knossian Palace Society in the Second Half of the 15th Century B.C.: Minoan or Mycenaean?, in: O. Krzyszkowska - L. Nixon (Hgg.), Minoan Society. Proceedings of the Cambridge Colloquium 1981 (Bristol 1983) 217-236.

\section{Niemeier 1986}

W.-D. Niemeier, Zur Deutung des Thronraumes im Palast von Knossos, AM 101, 1986, $63-95$.

\section{Palyvou 1987}

C. Palyvou, Circulatory Patterns in Minoan Architecture, in: R. Hägg - N. Marinatos (Hgg.), The Function of the Minoan Palaces (Stockholm 1987) 195-203.

\section{Panagiotopoulos 2006a}

D. Panagiotopoulos, Das minoische Stierspringen. Zur Semantik und Darstellung eines altägäischen Rituals, in: Y. Mylonopoulos - H. Röder (Hgg.), Archäologie und Ritual. Auf der Suche nach der rituellen Handlung in den antiken Kulturen Ägyptens und Griechenlands, Wiener Forschungen zur Archäologie (Wien 2006) 125-138.

\section{Panagiotopoulos 2006b}

D. Panagiotopoulos, Der minoische >Hof $<$ als Kulisse zeremonieller Handlung, in: J. Maran - C. Juwig - H. Schwengel - U. Thaler (Hgg.), Constructing Power. Architecture, Ideology and Social Practice (Hamburg 2006) 31-41.

\section{Pelon I983}

O. Pelon, Réflexions sur la fonction politique dans un palais crétois, in: O. Krzyszkowska L. Nixon (Hgg.), Minoan Society. Proceedings of the Cambridge Colloquium 1981 (Bristol 1983) 251-257. 


\section{Platon I97I}

N. Platon, Zakros. The Discovery of a Lost Palace of Ancient Crete (Amsterdam 1971).

\section{Reusch 1958}

H. Reusch, Zum Wandschmuck des Thronsaales in Knossos, in: E. Grumach (Hg.), Minoica. Festschrift zum 8o. Geburtstag von Johannes Sundwall (Berlin 1958) 334-348.

\section{Schiering 1960}

W. Schiering, Steine und Malerei in der minoischen Kunst, JdI 75, 1960, 17-36.

\section{Schroer 2006}

M. Schroer, Räume, Orte, Grenzen. Auf dem Weg zu einer Soziologie des Raums (Frankfurt a. M. 2006).

\section{Shaw 1995}

M. C. Shaw, Bull Leaping Frescoes at Knossos and Their Influence on the Tell el Dabca Murals, Ägypten und Levante 5, 1995, 91-120.

\section{Sikla 2003}

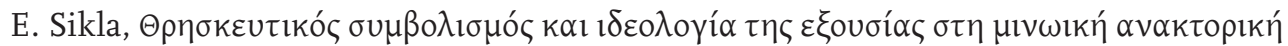

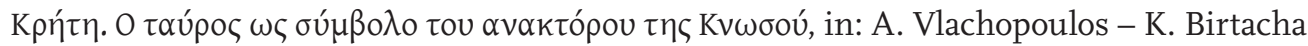

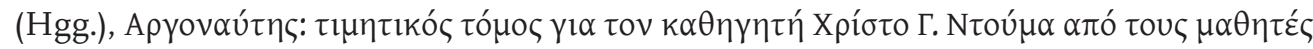

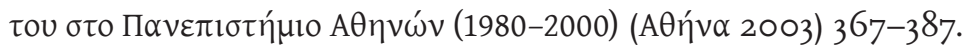

\section{Zanker 2000}

P. Zanker, Bild-Räume und Betrachter im kaiserzeitlichen Rom, in: A. H. Borbein T. Hölscher - P. Zanker (Hgg.), Klassische Archäologie. Eine Einführung (Berlin 2000) $205-226$.

\section{Zeimbekis 2006}

M. Zeimbekis, Grappling with the Bull: a Reappraisal of Bull and Cattle-Related Ritual

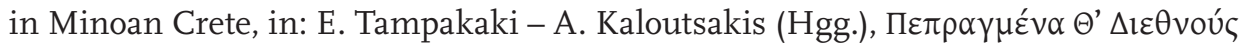

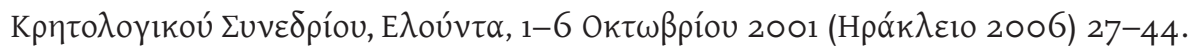

\section{Abbildungsnachweis}

Abb. 1: Anfertigung U. Günkel-Maschek nach Hood - Taylor 1981, Plan; Abb. 2: Anfertigung U. Günkel-Maschek nach Evans 1928, 672-679 Abb. 427-429; 719724 Abb. 450; Abb. 3: Umzeichnung U. Günkel-Maschek nach Evans 1928, Beil. XXVXXVII; Abb. 4: Anfertigung U. Günkel-Maschek nach Evans 1935, 905-921 Abb. 882. 884. 885. 890. 895 Taf. XXXII. XXXIII; Hood - Taylor 1981, Plan; Cameron 1987, 323 Abb. 7 . 